Original Article

\title{
Fibulin-5 Protein Is Reduced in the Lung of Patients with Spontaneous Pneumothorax Who Are Under 25 Years Old
}

\author{
Yoshimitsu Hirai, MD ${ }^{1,2}$ Yasuteru Muragaki, $\mathrm{MD}, \mathrm{PhD},{ }^{2}$ Shunji Itoh, $\mathrm{PhD},{ }^{2}$ \\ Kosuke Oikawa, $\mathrm{PhD},{ }^{2}$ Masanobu Juri, $\mathrm{MD}, \mathrm{PhD},{ }^{3}$ Tomohiro Kondo, $\mathrm{MD}, \mathrm{PhD},{ }^{4}$ and \\ Yoshitaka Okamura, $\mathrm{MD}, \mathrm{PhD}^{1}$
}

\begin{abstract}
Purpose: To clarify whether fibulins-5 is associated with primary spontaneous pneumothorax (PSP) in young PSP patients.

Methods: Forty-six surgically resected, fresh lung specimens were used. Patients were divided into 3 groups: younger than 25 years with pneumothorax (group Y), 25 years or older with pneumothorax (group $\mathrm{O}$ ), and without pneumothorax (group C). Chest X-ray, computed tomography data, height/width ratio $(\mathrm{H} / \mathrm{W})$ and anteroposterior/transverse diameter ratio (a/ b) were measured. Elastica van Gieson staining and immunofluorescence staining for fibulin-5 were performed. Fibulin-5 mRNA expression and protein levels were measured by real-time PCR and western blotting. Direct sequences of the fibulin-5 gene in PSP patients were performed.

Results: The mean $\mathrm{H} / \mathrm{W}$ ratio in group $\mathrm{Y}$ was significantly larger than that in the other groups $(p<0.01)$. The mean $a / b$ ratio in group $Y$ was significantly smaller than that in the other groups $(p=0.02)$. Fibulin-5 mRNA expression was not significantly different among the groups $(p=0.64)$. The relative intensity of fibulin-5 protein in group $Y$ was significantly lower than that in group $\mathrm{O}(\mathrm{p}=\mathbf{0 . 0 0 6})$, with no significant differences between groups $\mathrm{O}$ and $\mathbf{C}(\mathbf{p}=\mathbf{0 . 1 4})$.
\end{abstract}

Conclusions: We showed that fibulin-5 is reduced in patients with PSP who are younger than 25 years.

Keywords: fibulin-5, pneumothorax, bulla, Western blot, real-time reverse transcriptase polymerase chain reaction

\section{Introduction}

A high incidence of pneumothorax is a relatively common problem in Japan. Pneumothorax is defined as the presence of air in the pleural space. From a clinical stand point, spontaneous pneumothorax is classified as primary or secondary. ${ }^{1)}$ Primary spontaneous pneumothorax (PSP) is defined as pneumothorax occurring in patients with no underlying pulmonary disease. PSP commonly occurs in young, thin males. PSP has an incidence of 7.4-8 cases (age-adjusted incidence) and 1.2-6 cases/100000 population per year in males and females, respectively. ${ }^{2}$

Received: August 8, 2011; Accepted: October 18, 2011

Corresponding author: Yoshimitsu Hirai, MD. Department of Thoracic and Cardiovascular Surgery, Wakayama Medical University, 811-1 Kimiidera, Wakayama, Wakayama 641-8509, Japan

Email: mitsu@wakayama-med.ac.jp

(C)2012 The Editorial Committee of Annals of Thoracic and Cardiovascular Surgery. All rights reserved. 
Secondary spontaneous pneumothorax (SSP) usually occurs in older people with underlying pulmonary disease, such as chronic obstructive lung disease. SSP also occurs in patients with congenital connective tissue disorders such as Marfan syndrome ${ }^{3)}$ and Ehlers-Danlos Syndrome. ${ }^{4}$

PSP is usually caused by rupture of pleural blebs or bullae. ${ }^{5)}$ However, the exact pathogenesis of blebs or bullae and the reason why PSP frequently occurs in young, thin males are still unknown. Furthermore, in the clinical situation, many young patients who have no apparent risk factors, such as smoking, suffer from this disease.

The fibulin family is a group of widely expressed extracellular matrix $(\mathrm{ECM})$ proteins localized in the basement membrane, stroma, and ECM fibers. ${ }^{6}$ They mediate cell-to-cell and cell-to-matrix communication and provide organization and stabilization to ECM structures during organogenesis and vasculogenesis. ${ }^{7}$ Fibulin-5 is a recently identified fibulin family. ${ }^{8}$ Fibulin-5 contributes to the formation of elastic fibers by binding to structural components including tropoelastin and fibrillin- $1 .{ }^{9)}$ Studies in fibulin- 5 deficient mice have shown that fibulin-5 is necessary for the biosynthesis of elastic fibers. ${ }^{10)}$ Fibulin-5 is thought to be an adaptor for binding to microfibrils for the formation of elastic fibers. ${ }^{11)}$

In this study, we hypothesized that PSP in young patients is induced by previously unknown causes, which is different from SSP. We also hypothesized that fibulins-5 is associated with PSP in young PSP patients. We investigated the gene expression, protein, and tissue localization of fibulins-5 in patients with spontaneous pneumothorax patients and controls. Furthermore, we also analyzed clinical data on the shape of the thorax to reveal features of young PSP patients.

\section{Materials and Methods}

\section{Patients}

Surgically resected fresh lung specimens were obtained from 36 patients with pneumothorax and 6 patients with metastatic colorectal cancer as controls at Saiseikai Wakayama Hospital from June 2009 to September 2010. Four specimens were also obtained as controls when administrative autopsy was performed in our institute. Immediately after surgery, samples were snap-frozen in liquid nitrogen and stored at approximately $-80^{\circ} \mathrm{C}$. To analyze young PSP patients, patients with pneumothorax were divided into 2 groups. Patients younger than 25 years with pneumothorax were considered as the young

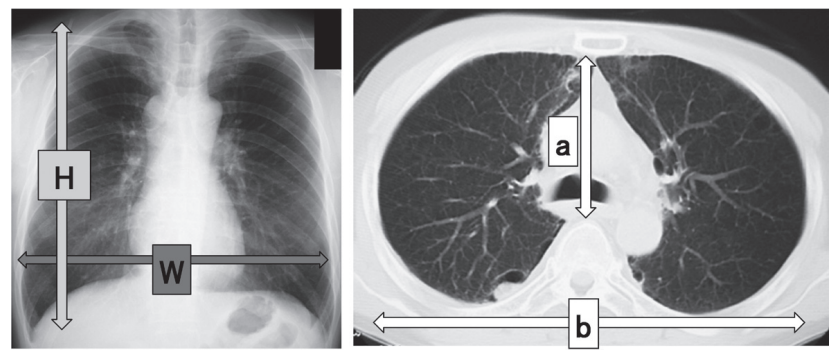

Fig. 1 a: Chest X-ray showing an AP view. The H/W ratio was calculated as height $(\mathrm{H})$ divided by a $\mid \mathrm{b}$ width (W).

b: Chest CT scan. The b/a ratio in the CT image was calculated as the anteroposterior diameter (a) divided by the transverse diameter (b) at the level of the first carina.

group ( $Y$ group, $n=14$ ), those aged 25 years or older with pneumothorax were considered as the old group (O group, $\mathrm{n}=22$ ). Patients without pneumothorax were also defined as the control group ( $\mathrm{C}$ group, $\mathrm{n}=10$ ). The study was approved by the ethics committee of Saiseikai Wakayama Hospital, and written informed consent was obtained from each patient.

\section{Clinical data analysis for chest deformity}

The anteroposterior (AP) view in chest X-ray and computed tomography (CT) scans was used to evaluate chest deformity of patients. The H/W ratio was calculated as height $(\mathrm{H})$ divided by width $(\mathrm{W})$ in the chest X-ray AP view (Fig. 1a). The b/a ratio in the $\mathrm{CT}$ image was calculated as the anteroposterior diameter (a) divided by the transverse diameter (b) at the level of the first carina (Fig. 1b). Height and weight data were also obtained.

\section{Visualization of elastin}

Elastin was visualized in paraffin section $(5 \mu \mathrm{m})$ by elastica van Gieson staining (Fig. 2a and 2b).

\section{Immunofluorescence staining for fibulin-5}

Tissue sections were deparaffinized and rehydrated through $100 \%, 95 \%$ and $90 \%$ ethanol solutions. For antigen retrieval, the slides were heated in a hot water bath for $15 \mathrm{~min}$ at $95^{\circ} \mathrm{C}$ followed by a 20-min cool-down period at room temperature. The sections were incubated with an anti-fibulin-5 polyclonal antibody (1:500, SC30170, Santa Cruz, CA) for $60 \mathrm{~min}$ at room temperature. FITC-anti-rabbit IgG (Sigma, St. Louis, MI) was used as a secondary antibody (Fig. 2c and $\mathbf{2 d}$ ). 

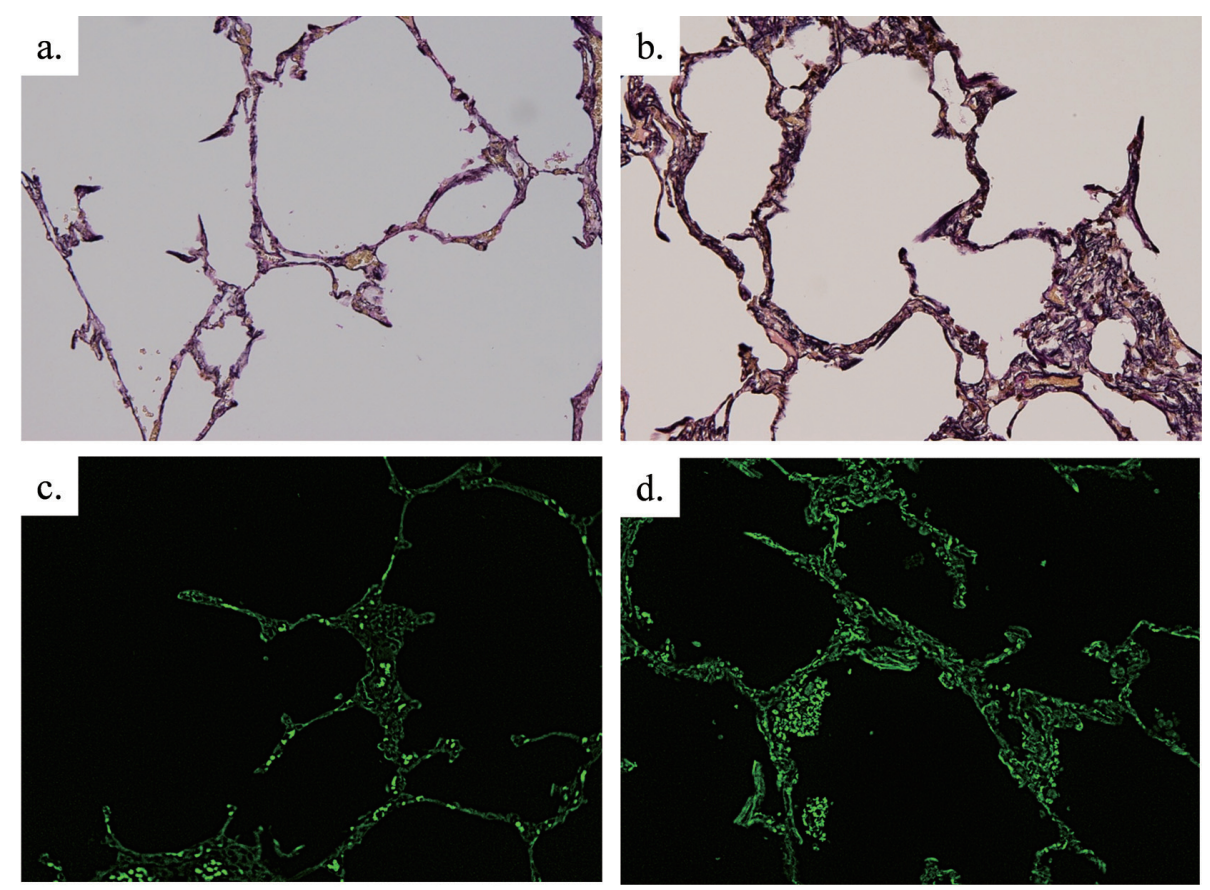

Fig. 2 Visualization of elastin and co-localization with fibulin-5. Elastica van Gieson staining (upper) and immunofluorescence staining for fibulin-5 (lower) are shown. a and c are the same samples in group Y. b and $\mathrm{d}$ are the same samples in group $\mathrm{O}$.

\section{Real-time RT-PCR}

After the frozen samples were homogenized, total RNA was extracted by Trizol reagent (Invitrogen, Carlsbad, CA). Reverse transcription to cDNA was performed using Superscript ${ }^{\mathrm{TM}}$ RNase H-Reverse Transcriptase (Invitrogen), and random oligonucleotide primers, synthesized according to the standardized protocol, were provided by the manufacturer.

To quantify the levels of mRNA encoding fibulin-5, the Applied Biosystems 7500 Real-Time PCR system (Applied Biosystems, Foster City, CA) was employed. GAPDH was used as an internal standard. All determinations were analyzed in triplicate in TaqMan ${ }^{\circledR}$ Universal Master Mix (Applied Biosystems) on 96-well optical PCR plates. Appropriate primers and probes were purchased from commercially available TaqMan gene expression assays (fibulin-5: Hs00197964_m1, GAPDH: Hs99999905_m1, Applied Biosystems). The intensities of expression of interest were expressed relative to the GAPDH intensities from the same sample.

\section{Western blot analysis}

For protein analysis, $100 \mathrm{mg}$ of tissue were homogenized and lysed in $500 \mu \mathrm{g}$ sample buffer $(0.125 \mathrm{ml} / 1$ Tris$\mathrm{HCl}$ [PH 6.8], 4\% SDS, 20\% glycerol, 2\% mercaptoetha- nol, and $0.03 \mathrm{mmol} / 1$ bromophenol blue). Five micrograms of protein from each sample were fractionated by $10 \%$ SDS polyacrylamide gel electrophoresis and transferred onto a nitrocellulose membrane (Biorad Laboratories, Richmond, CA). The membrane was blocked with phosphate-buffered saline (PBS), containing 5\% nonfat dry milk for $1 \mathrm{~h}$ at room temperature, and incubated overnight at $4{ }^{\circ} \mathrm{C}$ with primary antibodies directed against fibulin-5 (1:1000, SC-30170, Santa Cruz) and $\beta$-actin (1:1500, AC15, Sigma). After washing 3 times with PBS, the membrane was incubated in horseradish-peroxidaseconjugated secondary antibodies at room temperature for $1 \mathrm{~h}$. Bands were developed with the ECL plus detection system (Amersham Bioscience, Buckinghamshire, UK) and quantified by Scion image software (Scion Corp., MD). The intensities of bands were expressed relative to the $\beta$-actin intensities from the same sample.

\section{Statistical analysis}

All the values were calculated as the mean \pm standard deviation (minimum-maximum). Results were subjected to statistical analysis using the $\chi^{2}$ test and one-factor ANOVA. Values of $\mathrm{p}<0.05$ were considered to be significant. 
Table 1 Patient characteristics and clinical data in each group

\begin{tabular}{lcccc}
\hline & $\mathrm{Y}$ & $\mathrm{O}$ & $\mathrm{C}$ & $\mathrm{p}$-value (Y vs O) \\
\hline Patients characteristics & & & & \\
$\begin{array}{l}\text { Number of cases } \\
\text { Age (years) }\end{array}$ & 14 & 22 & 10 & \\
Sex & $19(16-24)$ & $46(27-72)$ & $60(14-79)$ & $<0.01$ \\
Male & 13 & 22 & 3 & 0.78 \\
Female & 1 & 0 & 7 & \\
Smoking status & & & & \\
smoker & 10 & 0 & 3 & $<0.01$ \\
non-smoker & 0 & 0 & 2 & \\
uncertain & 1.71 & 1.72 & 1.5 & 0.51 \\
Clinical data & 56 & 61.5 & 57 & 0.045 \\
Hight (m) & 1.19 & 1 & 0.99 & $<0.01$ \\
Weight (kg) & 0.3 & 0.34 & 0.4 & 0.22 \\
H/W ratio & & & & \\
a/b ratio & & & & \\
\hline
\end{tabular}

\section{Results}

\section{Patients' characteristics and clinical data analysis}

Patients' characteristics and clinical data of each group are summarized in Table 1. Thirty-five of 36 (97\%) patients with pneumothorax were males. All patients in group $\mathrm{O}$ were smokers, which is in contrast to 4 of 14 (28.6\%) smokers in group Y. There was no significant difference in height between group $\mathrm{Y}$ and $\mathrm{O}(\mathrm{p}=0.51)$. The mean weight in group $\mathrm{Y}$ was significantly smaller than that in group $\mathrm{O}(\mathrm{p}=0.045)$. The mean $\mathrm{H} / \mathrm{W}$ ratio in group $\mathrm{Y}$ was significantly larger than group $\mathrm{O}(\mathrm{p}<0.01)$. There was no significant difference in $\mathrm{a} / \mathrm{b}$ ratio between group $\mathrm{Y}$ and $\mathrm{O}(\mathrm{p}=0.22)$.

\section{Localization of fibulin-5}

Elastica van Gieson and immunofluorescence staining for fibulin-5 and elastin are shown in Fig. 2. Fibulin-5 was co-localized with elastic fibers. No apparent differences were observed between groups $\mathrm{Y}$ and $\mathrm{O}$.

\section{Messenger RNA expression analysis}

The distribution of relative fibulin-5 expression is shown in Fig. 3. Median relative intensities of fibulin-5/ GAPDH in groups $\mathrm{Y}, \mathrm{O}$, and $\mathrm{C}$ were $0.82 \pm 0.43,0.90 \pm$ 0.41 , and $0.76 \pm 0.27$, respectively. Fibulin -5 mRNA expression was not significantly different between groups $\mathrm{Y}$ and $\mathrm{O}(\mathrm{p}=0.97)$.

\section{Western blot analysis}

The distribution of relative intensities of fibulin-5 is shown in Fig. 4. Median relative intensities in group $\mathrm{Y}, \mathrm{O}$, and $\mathrm{C}$ were $1.10 \pm 0.44,1.64 \pm 0.58$, and $2.05 \pm 0.98$, re-

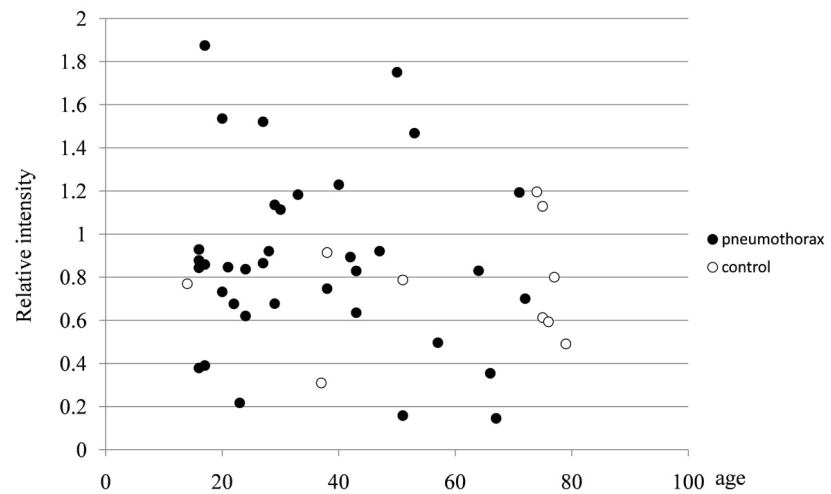

Fig. 3 Distribution of relative fibulin-5 expression.

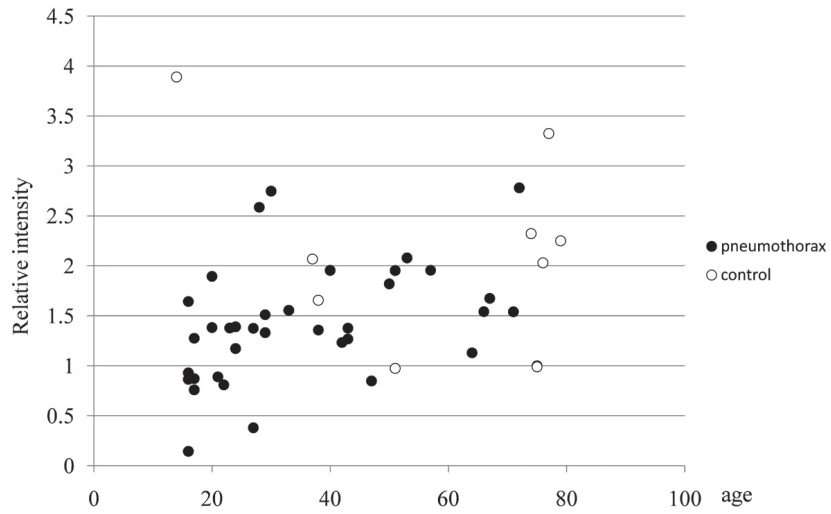

Fig. 4 Distribution of relative intensities of fibulin-5.

spectively. The relative intensity in group $\mathrm{Y}$ was significantly lower than group $\mathrm{O}(\mathrm{p}=0.006)$.

\section{Discussion}

PSP typically occurs in tall and thin males between 
the ages of 10 and 30 years and rarely occurs in persons over the age of 40 years. ${ }^{12)}$ Cigarette smoking increases the risk of PSP in men by as much as a factor of 20 in a dose-dependent manner. ${ }^{13)}$ Although patients with PSP do not have apparent lung disease, subpleural bullae are found in $48 \%$ to $100 \%$ of patients during video-assisted thoracoscopic surgery. ${ }^{5}$ However, the mechanism of bulla formation remains unclear. It is reported that the development of blebs and bullae appear to be linked to a variety of factors, including distal airway inflammation, ${ }^{14)}$ hereditary predisposition, ${ }^{15)}$ ectomorphic physiognomy with more negative intrapleural pressures ${ }^{16)}$ and apical ischemia, ${ }^{17)}$ and abnormal connective tissue. ${ }^{18,19)}$ However, there are many patients with PSP without these risk factors. In these cases, the true cause of pneumothorax is still unknown.

The fibulins are a relatively recently recognized family of extracellular proteins that are widely expressed in the basement membranes of epithelia and blood vessels.7) All fibulins have binding sites for other basement membrane proteins, such as fibrillin, fibronectin, proteoglycans, integrins, and tropoelastin. Fibulin-5 appears to be essential for the polymerization of elastin, because in mice and humans without functional copies of the fibulins-5 gene, tropoelastin is synthesized but is not assembled into its mature elastin fibrils. ${ }^{9,20,21)}$ Fibulin- $5^{-/}$mice have loose skin hanging in folds, a short nose, and large cheeks. In addition to these changes, histological observation of the lung tissue revealed disruption of alveolar structure in adult fibulin- $5^{-/-}$lungs, which were most severe in the peripheral regions. ${ }^{9,20)}$

We hypothesized that fibulin-5 reduction leads to fragileness of lung tissue and development of blebs and bullae. First, we analyzed the clinical data to determine features of young patients with PSP. There were no apparent risk factors in patients with PSP except for smoking. All patients with pneumothorax who were older than 25 years were smokers. To determine these features and distinguish the influence of risk factors from age, patients were divided into 2 groups: younger than 25 years (group Y) and 25 years or older (group O). Our clinical data analysis showed that patients in group $\mathrm{Y}$ had a thin thorax compared with those in group $\mathrm{O}$, although height and $\mathrm{a} / \mathrm{b}$ ratio were not different between pneumothorax patients. These results suggested that young PSP patients have a long thorax rather than a thin thorax.

We then examined fibulin-5 protein levels and mRNA expression in pneumothorax patients and controls. We showed that fubulin-5 protein levels in group Y patients were significantly lower than those in group $\mathrm{O}$ and $\mathrm{C}$ patients. On the other hand, fibulin-5 protein levels were not significantly different when they were compared between group $\mathrm{O}$ and $\mathrm{C}$ patients $(\mathrm{p}=0.14)$. There was only 1 patient younger than 25 years in group $\mathrm{C}$, and the protein level of fibulin-5 in this 14-years-old patient was high. These findings may indicate an association between fibulin-5 reduction and PSP in patients younger than 25 years. Since fibulin-5 is essential for elastogenesis, this indicates that the reduction of fibulin-5 protein results in a corresponding reduction in elastogenesis. However, mRNA expression of fibulin-5 was not significantly different among the groups. It is unclear why fibulin-5 protein was reduced in group $\mathrm{Y}$ without reduction of fibulin-5 mRNA expression.

Age-related macular degeneration is the most common cause of irreversible visual loss in the Western world. ${ }^{22,23)}$ Recently, missense mutations in fibulin-5 have been reported in association with age-related macular degeneration. ${ }^{24)}$ Lotery et al. revealed that fibulin-5 secretion was significantly reduced for 4 age-related macular degeneration (p.G412E, p.G267S, p.I169 T, and p.Q124P) and 2 cutis laxa (p.S227P, p.C217R) mutations. ${ }^{25)}$ They showed that some missense mutations lead to decreased fibulin-5 secretion with a possible corresponding reduction in elastinogenesis. To clarify the mechanism of reduction in fibulin-5 protein, we performed sequence analysis of fibulin-5. However, no sequence variations in fibuoin-5 were found in pneumothorax patients.

In this study, there are some limitations with regard to samples. It was ethically difficult to obtain young healthy lung samples. Almost all patients who required surgery had pneumothorax or some congenital disorder and they were not suitable as controls. Therefore, we could obtain only 1 sample from a young healthy patient in this study.

In conclusion, we showed fibulin-5 reduction in patients with PSP younger than 25 years old. This is the first report of the association between PSP and fibulin-5. A long and thin thorax rather than a tall stature was also observed in young PSP patients. However, our results failed to clarify how fibulin-5 protein was reduced and the association between fibulin-5 reduction and chest deformity. Further investigation, e.g., investigation using animal models, will be required.

\section{Acknowledgments}

We are grateful to Dr. Yoshida, Dr. Nakai, Dr. Nakamura, Dr. Iwahashi, and Dr. Toguchi at Saiseikai 
Wakayama Hospital for help with data collection. This study was also supported by clinical laboratory technicians at Saiseikai Wakayama Hospital, who helped with the accumulation of clinical samples.

\section{Disclosure Statement}

The authors report to Annals of Thoracic and Cardiovascular Surgery that no potential conflicts of interest exist with any companies/organizations whose products or services may be discussed in this article.

\section{References}

1) Baumann MH, Noppen M. Pneumothorax. Respirology 2004; 9: 157-64.

2) Bense L, Eklund G, Wiman LG. Smoking and the increased risk of contracting spontaneous pneumothorax. Chest 1987; 92: 1009-12.

3) Wood JR, Bellamy D, Child AH, et al. Pulmonary disease in patients with Marfan syndrome. Thorax 1984; 39: 780-4.

4) Pepin M, Schwarze U, Superti-Furga A, et al. Clinical and genetic features of Ehlers-Danlos syndrome type IV, the vascular type. N Engl J Med 2000; 342: 673-80.

5) Schramel FM, Postmus PE, Vanderschueren RG. Current aspects of spontaneous pneumothorax. Eur Respir J 1997; 10: 1372-9.

6) Argraves WS, Greene LM, Cooley MA, et al. Fibulins: physiological and disease perspectives. EMBO Rep 2003; 4: 1127-31.

7) Timpl R, Sasaki T, Kostka G, et al. Fibulins: a versatile family of extracellular matrix proteins. Nat Rev Mol Cell Biol 2003; 4: 479-89.

8) Albig AR, Schiemann WP. Fibulin-5 function during tumorigenesis. Future Oncol 2005; 1: 23-35.

9) Yanagisawa H, Davis EC, Starcher BC, et al. Fibulin-5 is an elastin-binding protein essential for elastic fibre development in vivo. Nature 2002; 415: 168-71.

10) Zheng Q, Choi J, Rouleau L, et al. Normal wound healing in mice deficient for fibulin-5, an elastin binding protein essential for dermal elastic fiber assembly. J Invest Dermatol 2006; 126: 2707-14. Epub 2006 Aug 10.

11) Hirai M, Ohbayashi T, Horiguchi M, et al. Fibulin-5/ DANCE has an elastogenic organizer activity that is abrogated by proteolytic cleavage in vivo. J Cell Biol 2007; 176: 1061-71. Epub 2007 Mar 19.

12) Primrose WR. Spontaneous pneumothorax: a retrospective review of aetiology, pathogenesis and management. Scott Med J 1984; 29: 15-20.

13) Gobbel WG Jr., Rhea WG Jr., Nelson IA, et al. Spontaneous pneumothorax. J Thorac Cardiovasc Surg 1963; 46: $331-45$.

14) Noppen M, De Keukeleire T. Pneumothorax. Respiration 2008; 76: 121-7.

15) Morrison PJ, Lowry RC, Nevin NC. Familial primary spontaneous pneumothorax consistent with true autosomal dominant inheritance. Thorax 1998; 53: 151-2.

16) Fujino $S$, Inoue $S$, Tezuka $N$, et al. Physical development of surgically treated patients with primary spontaneous pneumothorax. Chest 1999; 116: 899-902.

17) Withers JN, Fishback ME, Kiehl PV, et al. Spontaneous pneumothorax. Suggested etiology and comparison of treatment methods. Am J Surg 1964; 108: 772-6.

18) Han S, Sakinci U, Kose SK, et al. The relationship between aluminum and spontaneous pneumothorax; treatment, prognosis, follow-up? Interact Cardiovasc Thorac Surg 2004; 3: 79-82.

19) Leo F, Venissac N, Drici MD, et al. Aluminium and primary spontaneous pneumothorax. A suggestive but unconfirmed hypothesis. Interact Cardiovasc Thorac Surg 2005; 4: 21-2. Epub 2004 Dec 17.

20) Nakamura T, Lozano PR, Ikeda Y, et al. Fibulin-5/ DANCE is essential for elastogenesis in vivo. Nature 2002; 415: 171-5.

21) Loeys B, Van Maldergem L, Mortier G, et al. Homozygosity for a missense mutation in fibulin-5 (FBLN5) results in a severe form of cutis laxa. Hum Mol Genet 2002; 11: 2113-8.

22) Klaver CC, Wolfs RC, Vingerling JR, et al. Age-specific prevalence and causes of blindness and visual impairment in an older population: the Rotterdam Study. Arch Ophthalmol 1998; 116: 653-8.

23) Tielsch JM, Javitt JC, Coleman A, et al. The prevalence of blindness and visual impairment among nursing home residents in Baltimore. N Engl J Med 1995; 332: 1205-9.

24) Stone EM, Braun TA, Russell SR, et al. Missense variations in the fibulin 5 gene and age-related macular degeneration. N Engl J Med 2004; 351: 346-53.

25)Lotery AJ, Baas D, Ridley C, et al. Reduced secretion of fibulin 5 in age-related macular degeneration and cutis laxa. Hum Mutat 2006; 27: 568-74. 